\title{
AUGMENTED REALITY AND INTERNET OF THINGS - IMPLEMENTATION IN PROJECTS BY USING SIMPLIFIED ROBOTIC MODELS
}

\author{
Zorica A. Dodevska ${ }^{1}$, Vladimir Kvrgić ${ }^{2}$, Velimir Štavljanin ${ }^{3}$ \\ ${ }^{I}$ Research and Development Institute Lola Ltd, Serbia \\ ${ }^{2}$ Institute Mihajlo Pupin (IMP), Serbia \\ ${ }^{3}$ Faculty of Organizational Sciences, University of Belgrade, Serbia
}

\begin{abstract}
Augmented reality (AR) and internet of things (IoT), technologies that have recently expanded in various fields of theory, scientific researches, and business practice, provide innovative approach for development of projects and their implementation. In this paper, one approach of the implementation is undertaken as an experimental use case, applied on a simplified robotic model of centrifuge for pilot training and flight simulation. An AR/IoT mobile app is designed to be developed in two phases. The first phase is finished, and the second one is under development. The app contains four main parts with the following functions: presentation, monitor/control, pilot testing, and analytics. The system and possibilities of the implementation of the app in the belonging project are explained in the paper. This use case indicates that AR/IoT apps can be developed by using simplified robotic models and later transferred to the real objects that are subject of research projects, even before their actual construction.
\end{abstract}

Key words: Augmented reality, internet of things, project, simplified robotic model.

\section{INTRODUCTION}

Robotic systems mediate information among people in the sense that people both embed in and use knowledge through robots. Technologies greatly help in knowledge transfer. Especially, augmented reality (AR) and internet of things (IoT) proved to be successful in this process. Similar situation is about the acquisition of complex skills, where $\mathrm{AR}$ and IoT result in more productive trainings. It is possible thanks to multimedia communication which take place at many different levels. Therefore, AR, IoT, and underlying multimedia communication will be in the focus in this paper, particularly in the relation of the human-machine interaction. In this context, AR will be regarded as an interface to end-user, while all background communication among devices will be explained based on IoT concept.

In this paper the authors use AR and IoT and explain how the technologies work. Some possibilities of their application in training projects are examined, specifically in trainings specialized for flight. After that, the authors present an experimental use case with the aim to demonstrate implementation of AR and IoT on a simplified robotic model (SRM) that is a subject of a research project.

The broader idea is building functional AR and IoT systems, or parts of systems, that can be transferred to the real objects, with the necessary modification, calibration and testing.

In this paper, SRM of the centrifuge has been considered as an experimental use case. The ultimate goal in this work is to help the respondents to overcome strenuous trainings due to g-forces. This goal would be greatly supported through the use of AR and IoT on SRM. AR/IoT app is develop for SRM of centrifuge to present possibilities of its application. This solution points out differences in the case of using and not using these technologies.

Corresponding author. Email: zorica.dodevska@gmail.com

ISSN 2560-4961 (online)

(C) 2018 IPMA Serbia

doi: 10.18485/epmj.2018.8.2.4 


\section{AR AND IOT - INTERCONNECTED TECHNOLOGIES}

Augmented reality is experience and technology that provides superimposition or overlay of digital content over real-world environment in real time (Dodevska, \& Mihić, 2018; Dodevska, Mihić, \& Manasijević, 2018), as well as three main capabilities to end-users: visualization, instruction and guidance, and interaction (Porter \& Heppelmann, 2017).

In the literature, there are various criteria for categorization of $\mathrm{AR}$, and some of the most important are mentioned in Table 1.

Table 1: AR categorization depending on criteria

\begin{tabular}{|c|c|}
\hline Criteria & AR categorization \\
\hline $\begin{array}{l}\text { AR types (depending } \\
\text { on how AR technology } \\
\text { starts and works): }\end{array}$ & $\begin{array}{ll}\text { - } & \text { Marker-based AR } \\
\text { - } & \text { Markerless AR }\end{array}$ \\
\hline $\begin{array}{l}\text { AR interfaces } \\
\text { (depending on the way } \\
\text { of interaction between } \\
\text { end-user and AR app), } \\
\text { according to } \\
\text { Carmigniani et al. } \\
(2011) \text { : }\end{array}$ & $\begin{array}{ll}\text { - } & \text { Tangible interface } \\
\text { - } & \text { Collaborative } \\
\text { - } & \text { Hyterface } \\
\text { - } & \text { Mubrid interface } \\
& \text { interface }\end{array}$ \\
\hline $\begin{array}{l}\text { AR presentation } \\
\text { (depending on visual } \\
\text { display): }\end{array}$ & $\begin{array}{l}\text { - Video see-through } \\
\text { presentation } \\
\text { - Optical see- } \\
\text { through } \\
\text { presentation }\end{array}$ \\
\hline
\end{tabular}

$\mathrm{AR}$ as an interface to end-users is closely related with the following technologies (Dodevska, \& Filipović, 2018), as illustrated in Figure 1:

- Internet of things (IoT) - IoT provides connectivity and communication among "smart" devices including sensor data and multimedia content exchange which are important part of AR solutions;

- Virtual reality (VR) - AR adds virtual objects to real reality (RR) for its better understanding, and deals with these twocontrary concepts (RR and VR) on Milgram's Reality-Virtuality continuum
(Milgram, Takemura, Utsumi, \& Kishino, 1995);

- Global positioning system (GPS) markerless AR uses GPS data for positioning and tracking;

- Simultaneous localization and mapping (SLAM) - it can be used for markerless AR for more precise tracking, objects and scene recognition in unknown space, and under conditions where GPS signal is not available (for example, in zones with high radiation level, or on other celestial bodies);

- Artificial intelligence (AI) - AR apps can include software that uses machine learning, databases, and remote libraries for data processing, analyzing, and placing the right information to the end-user.

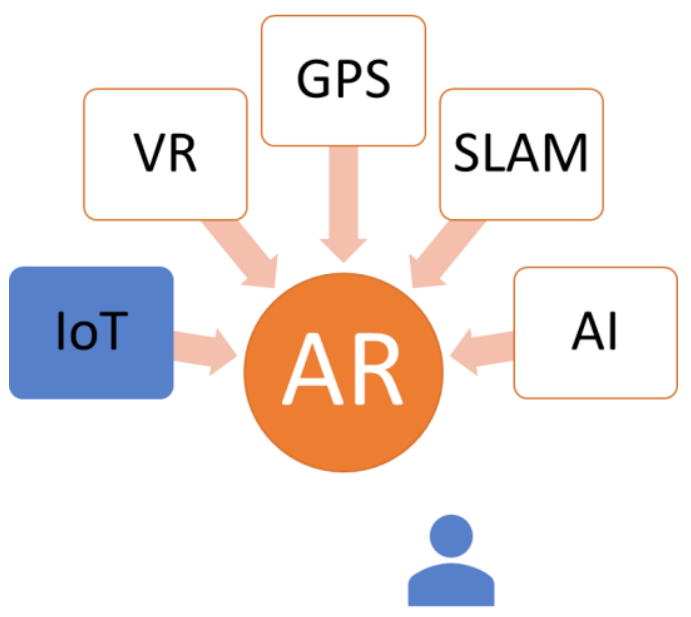

Figure 1: $A R$ as an interface to end-user

Internet is divided in two interweaved communities: internet of people - IoP and internet of things - IoT (PTC University, Heppelmann), and IoT becomes rapidly predominant. Smart connected products, with three main components: physical, "smart", and connectivity (Porter, \& Heppelmann, 2014), bring new values, possibilities, and increase complexity of systems. IoT represents a network of connected 'things', where 'thing' is a digital representation of physical object with unique identifier and capability to transmit data. IoT systems are complex having in the mind the number of connected things, their diversity (personal devices, home devices, vehicles, buildings, etc.), as well as the number and diversity of their connections.

AR and IoT are not necessarily connected technologies. However, their joint usage is an 
innovative way of efficient application of those technologies in AR/IoT apps. IoT provides information about devices via sensors in real time from the location and forward them to the AR part of the app. IoT part of the app accepts service requests from the AR part of the app (for example, they can be related to home temperature regulation, machine speed, information flow, security aspects of assets and objects). All this can be done either on the spot or from remote locations, thanks to sophisticated information exchange protocols between the object of interest and their edge devices from one side, and edge devices and cloud infrastructure on the other side (National Instruments, 2018). Once the data are received on the cloud, thanks to the software part of the app, they can be processed, analyzed and forwarded to the interested parties, no matter whether they are people or machines.

Communication modes between the hardware and software part of the appl can be one-way (for example, REST API can be used only for data collection) or two-way (for example, Edge Micro Server - EMS which allows collecting data from sensors as well as sending service requests from user apps no matter whether the service request is sent by people or machines).

\section{TRAININGS SPECIALIZED FOR FLIGHT}

Training \& Practice and Education \& Learning are significant $\mathrm{AR}$ roles in human activities regarding the number of articles in the last 30 years (Dodevska, \& Putnik, 2018). Training is also recognized as important AR role in defensive activities (Dodevska, et al., 2018). AR can be useful tool for training specialized for flights, both for initial training of respondents (usually according to a pre-set procedure) as well as for advanced training programs (which can include simulation of the real situation in the case of preparation for special tasks, military exercises, etc).
Head-mounted display (HMD) or screen can be used as a device for respondents in real centrifuge for:

- Monitoring and controlling of physical parameters (motion, g-force, etc) from the cockpit and from control room;

- Monitoring of health parameters, situation awareness, emotions and stress, loss of consciousness (LOC) during the spatial disorientation of pilots, etc.

- Monitoring of flight technique and performance of tasks in controlled conditions;

- Corrections in decisions and procedures during flight;

- Analytics of effectiveness of the mission's fulfilment, comparison with earlier results, etc.

The parameters of the movement of cockpit and the rotation of the centrifuge could be seen as in the real conditions in a plane in a combat situation.

\section{AR/ IOT SYSTEM FOR SIMPLIFIED ROBOTIC MODEL OF CENTRIFUGE}

A scheme of AR/IoT system of simplified robotic system is shown in Figure 2. Similarly, as in the system overview presented by Cheli, Sinapov, Danahy, \& Roger (2018), it contains the following components:

1. EV3 Robot Centrifuge - it represents the SRM environment;

2. LEGO ${ }^{\circledR}$ MINDSTORMS ${ }^{\circledR}$ EV3 Software and separated computer - the subsystem that drives SRM and pulls the sensor data;

3. ThingWorx Cloud Platform - it merges images from camera with IoT data, provides calculations and analytics, and mediates communication;

4. Mobile App - developed in Vuforia Studio (part of ThingWorx Cloud Platform) to enable AR/IoT user view and interaction through Vuforia View. 


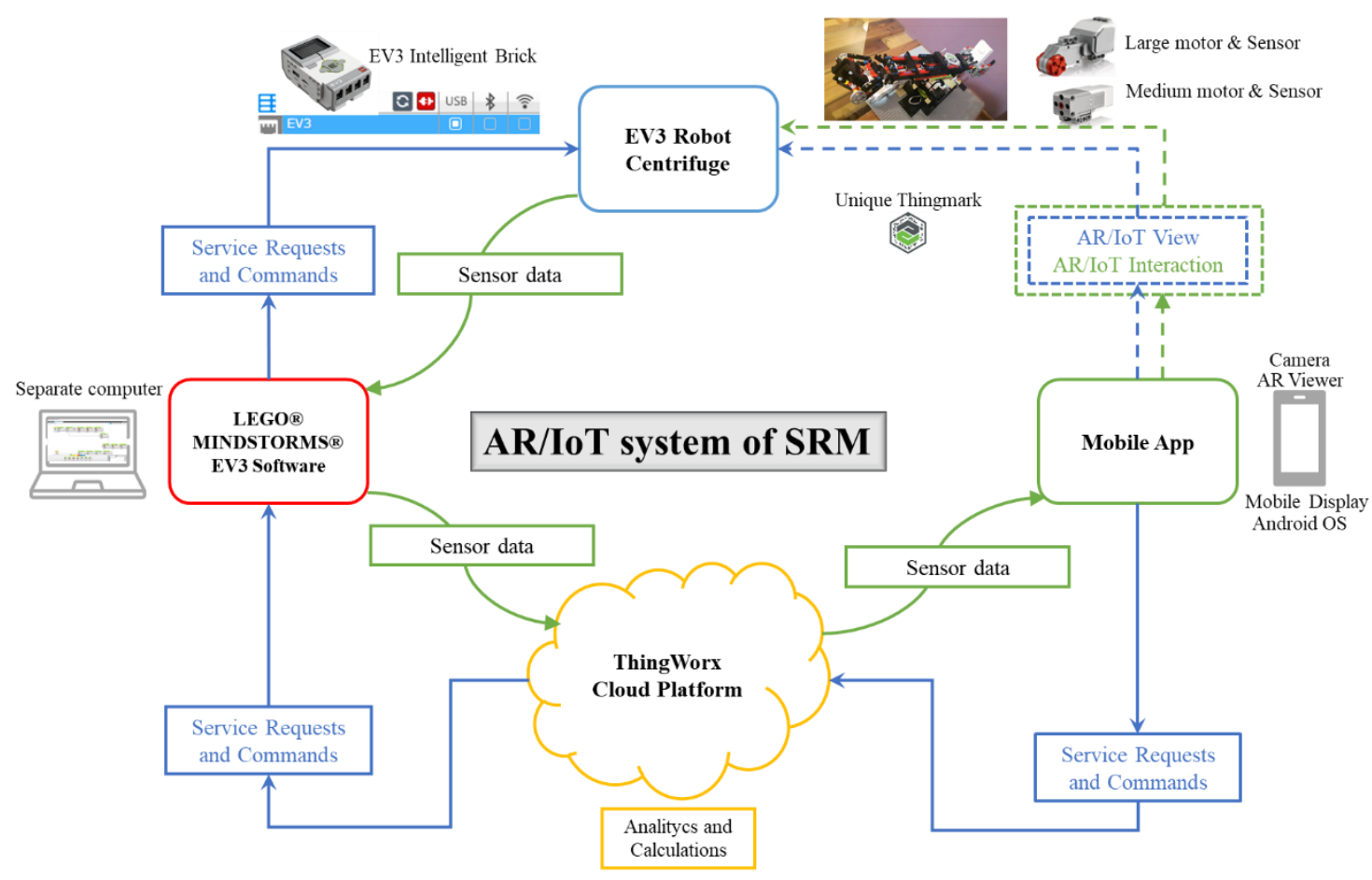

Figure 2: Scheme of AR/IoT system of simplified robotic system

\subsection{EV3 Robot Centrifuge}

The centrifuge has two rotational axes. Robotic arm rotates around the main axis (z-axis). This is the vertical (planetary) axis. Second rotational axis is pitch. It lies in the plane of the arm rotation, perpendicular to the main rotational axis. It is located in the cockpit. Simplified robotic model of centrifuge is presented in Figure 3.

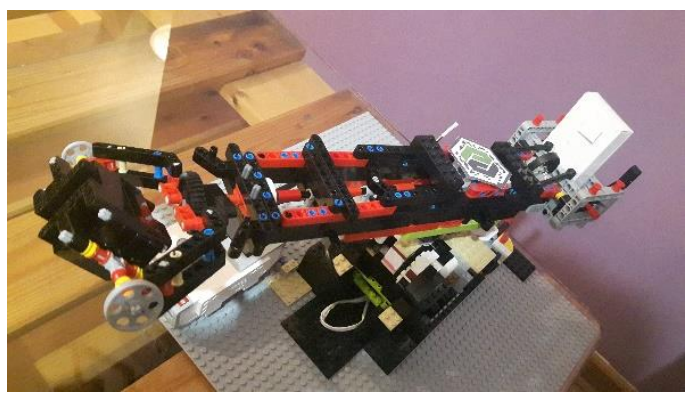

Figure 3: Simplified robotic model of centrifuge

All axes of rotation have independent control mechanism and can rotate in two directions.

Construction has been made using LEGO Mindstorms complete and several additional elements (one slip ring - 12 Wire (2A), additional LEGO building elements, counterweight placed on the contrary side of the cockpit). The length of the robot arm from the centre of the cockpit to the centre of the main axis of rotation (z-axis) equals $0,265 \mathrm{~m}$.

The main axis is powered by EV3 Large motor. The motor powers 36-tooth gear, which is connected to the motor by a vertical axle. This gear transmits the rotation to a 20-tooth gear, which is located at the main axis of rotation of the centrifuge. The large motor is connected to the A port of EV3 Intelligent Brick.

The pitch is powered by EV3 Medium motor. Since this is rotation of cockpit, in order to avoid entanglement of the cables, the connection of the medium motor with B port of EV3 Intelligent Brick is accomplished thought the slip ring.

All motors have sensors for measuring the number of rotations, which are based on incremental encoder.

\subsection{LEGO® MINDSTORMS® EV3 Software and Separated Computer}

LEGO ${ }^{\circledR}$ MINDSTORMS ${ }^{\circledR}$ EV3 Software and separated computer are used to write program that drives SRM. They also pull sensor data from SRM. Two program flows have been written, one for each axis of rotation (Figure 4). 


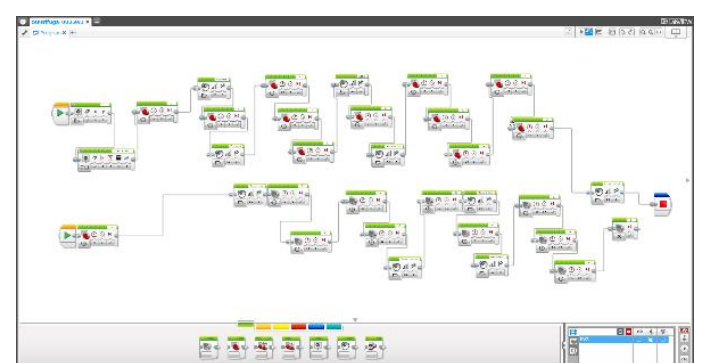

Figure 4: $L E G O \circledR$ MINDSTORMS ${ }^{\circledR}$ EV3 Software program for SRM

Separated computer is not the part of SRM. It is also separated from ThingWorx Cloud Platform and from the user mobile device.

\subsection{ThingWorx Cloud Platform}

ThingWorx Cloud Platform is a cloud-based development platform. Among others, it includes Vuforia Studio for AR development, ThingWorx Foundation (which includes EMS) for IoT part of the app for SRM, as well as analytics tools, that are used in this AR/IoT system.

Calculation of g-force of centrifuge is done at a constant rotation, in several steps:

1. For calculation of the acceleration of the centrifuge at a constant rotation the following formula is used:

$$
a_{c}=4 \pi^{2} r n^{2}
$$

where $r$ represents the length of the robot arm (in the case of z-axis it is $0.265 \mathrm{~m}$ ), and $n$ represents the number of rotations per second (RPS) of the motor. Calculation of acceleration is programmed at several constant rotational speeds.

2. In order to calculate g-force $(\mathrm{G})$, the following formula (according to Kvrgic, Vidakovic, Lutovac, Ferenc, \& Cvijanovic, 2014) is used:

$$
G=\frac{\sqrt{a_{\mathrm{c}}^{2}+a_{\mathrm{t}}^{2}+\mathrm{g}^{2}}}{\mathrm{~g}}
$$

where $g$ is $9.81 \mathrm{~m} / \mathrm{s}^{2}$ (the acceleration of gravity), while $a_{t}=0 \quad$ (tangential acceleration) for constant rotation.

3. In the case of z-axis, the number of $n$ is product of multiplication of RPS of the
EV3 Large motor and gear ratio that equals 1,8 (or $36 / 20$ ).

The g-force is rounded to two decimal places. The calculation is embedded in filters of Vuforia Studio widgets.

\subsection{Mobile App}

Development of the mobile app is planned in two phases. The first phase is completed and includes definition of the overall look, development of CSS design, making graphical elements (buttons, images, panels), development of the main views and functionalities. The second phase is under development and includes finishing of the Pilot/Test View and Analytic View, as well as the real time IoT communication by using EMS within ThingWorx Foundation.

\subsubsection{Configuration}

AR/IoT mobile app is titled AR/IoT app: Simplified Robotic Model of Centrifuge. It is configured to be started with ThingMark or Quick Response (QR) Code. The app could be started without ThingMark by using spatial tracking, but it is not suitable for this app because the data should be superimposed over the real-world robotic model.

The app is configured to be started in Vuforia View mobile app, by recognizing unique ThingMark directly or by QR code which should be scanned with Vuforia View (version 8.3.0 or later) to start it on a device. The navigation menu is obtained through Collapse Menu Button or "sandwich" menu. A simple 3D model of centrifuge is modelled and put as a placeholder within Vuforia Studio to help easier positioning of AR elements. The placeholder is not visible during the runtime.

In this project phase the app uses the portrait orientation of a mobile phones. In the future it is planned to make both portrait and landscape orientation for all mobile devices (phones and tablets).

A custom Cascading Style Sheets (CSS) is made for the whole app. The app is developed in Vuforia ${ }^{\mathrm{TM}}$ Studio version 8.3.7 (Copyright (C) 2018 PTC Inc). 


\subsubsection{Home View}

Home View contents welcoming title, a short description of the app, image showing a visual representation of the app, and navigation buttons for the main app views (Figure 5):

- Presentation View;

- Video View;

- Monitoring/Control View;

- Pilot/Test View;

- Analytic View.

In the Home View there is a button that links to a video that gives more information about project.

\section{1:05 SinSTPRed Robotic Model of Centrifuge}

$\equiv \quad$ Home View

\section{WELCOME TO AR/IOT APP}

AR/loT app is developed for simplified robotic model of centrifuge to present possibilities of the application of AR/loT in development of the project TR-35023.

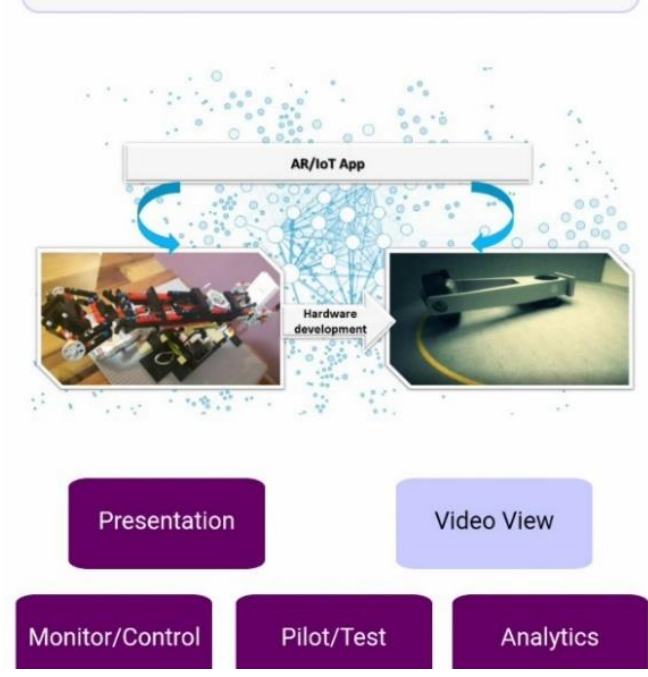

Figure 5: Home View

\subsubsection{Presentation View}

Presentation View includes four custom-made image buttons which link to the following AR elements in the app: specification, cockpit, $\mathrm{z}$ axis, pitch. All these elements are presented in the app by using 3D image panels with socalled billboard characteristics (they face to user all the time). Those panels contain the information about the main parts of the centrifuge. The panels are anchored within the scene with a ThingMark located at the top of $\mathrm{z}$ axis of the centrifuge. There are also three static labels which show position of cockpit and motors. It is worth to note that all those elements are arranged in $3 \mathrm{D}$ view so that they support 3D impression for the viewer: the farther element is positioned in behind of a closer object. Figure 6 shows a print screen of building 3D Presentation View in Vuforia Studio.

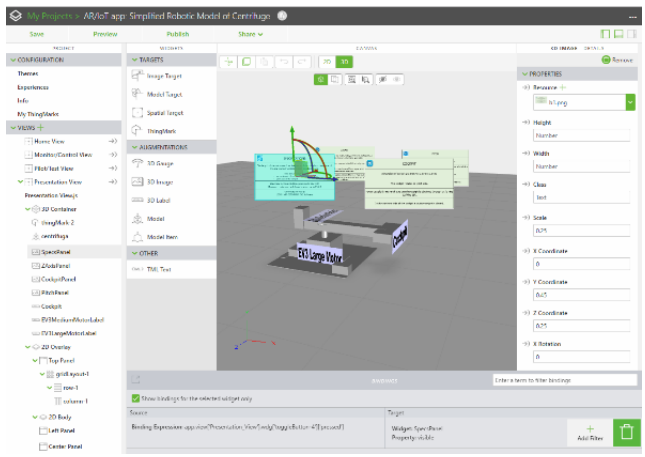

Figure 6: Building 3D Presentation View in Vuforia Studio

Presentation View from the mobile app is given in Figure 7.

\section{0:57m STOP Robotic Model of Centrifu... (-) ...}

\section{$\leftarrow$ Back Presentation View}

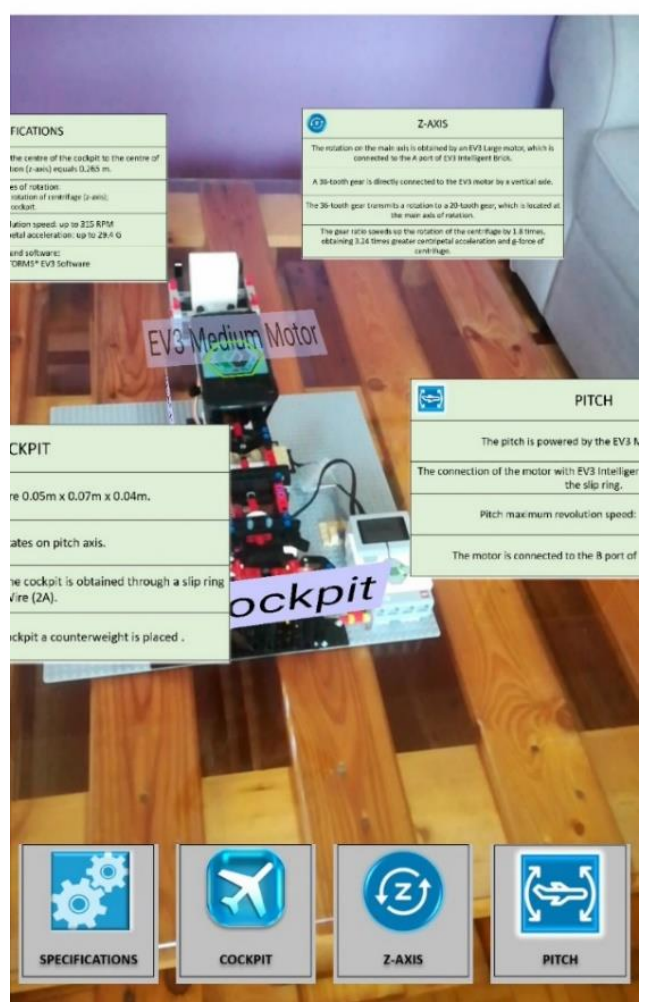

Figure 7: Presentation View 


\subsubsection{Monitor/Control View}

Monitor/Control View is one of the most complex parts of the app. It contains commands for monitoring the sensor data of centrifuge, and a command interface for controlling its motors. At the bottoms of the screen there are four custom image buttons. Each one of them shows several AR elements, some of them in 2D screen space and some of them in 3D space of the scene. Those groups of AR elements are: EV3 Large motor related elements to monitor and control the rotation around z-axis, EV3 Medium motor related elements to monitor and control the rotation around pitch (y-axis), cockpit related AR elements to monitor $\mathrm{g}$-force of z-axis and $\mathrm{y}$ axis, and elements to monitor RPS around $\mathrm{z}$ axis and y-axis (Figure 8).

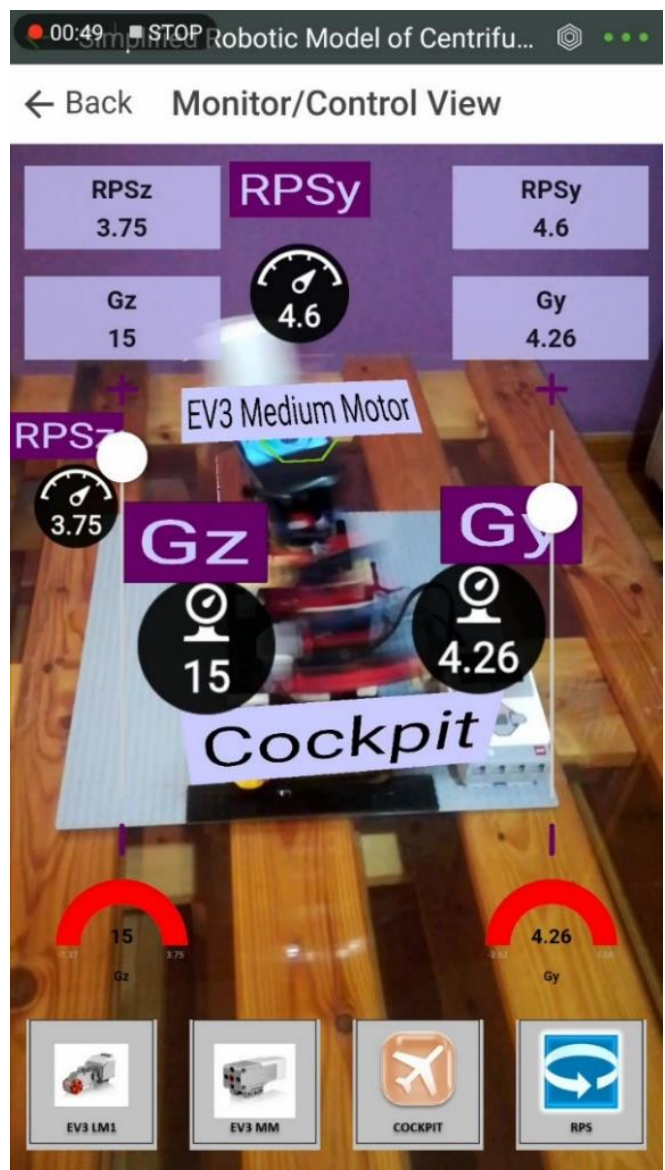

Figure 8: Monitor/Control View

In first phase, all monitor/control data are result of an educated simulation. The limits for rotation, and by that g-force, are calculated based on g-force limits planed for the real centrifuge in the project:

$\mathrm{G}_{\mathrm{y}} \in[-14 \mathrm{G}, 10 \mathrm{G}]$,
$\mathrm{G}_{\mathrm{z}} \in[-2 \mathrm{G}, 15 \mathrm{G}]$.

This means that limits for RPS of SRM are:

RPS $_{\mathrm{y}} \in[-8.34,7.05]$,

$\mathrm{RPS}_{z} \in[-1.37,3.75]$.

JavaScript Snippets are used for calculation of $g$-force. Here is an example for calculation of $\mathrm{g}$-force of z-axis:

return (value $>=0$ ? Number $((4 * 3.1416 *$ $3.1416 *$ value $*$ value $*$ 0.265)/9.81).toFixed(2)) : Number(((4) * $3.1416 * 3.1416 *$ value * value * 0.265)/9.81).toFixed(2)) *-1);

The press on cockpit and RPS buttons show instruments that monitor calculated $\mathrm{G}_{\mathrm{y}}$ and $\mathrm{RPS}_{\mathrm{y}}$, as well as $\mathrm{G}_{\mathrm{z}}$ and $\mathrm{RPS}_{\mathrm{z}}$. They are located in the 3D place of the simulation in the similar way as they will be placed in the real object.

The simulation of RPS and g-force is done by sliders with the mentioned limits. They are located in 2D screen space in the app together with some monitoring instruments.

In the bottom of the screen there are two gauges, each for one slider, that show red colour in the case of high g-force.

\subsubsection{Pilot/Test View}

Pilot/Test View is designed for a future version of this app. It will contain several tests similar for pilot testing in the real object. Some of them are: balancing the plane in the situation where a simulated flight is given from control room, tracking another plain, or dealing with swatches and instruments in the cockpit. Multidirectional AR pointing tasks under simulated hypergravity according to study of MarkovVetter, Zander, Latsch, \& Staadt, (2015) are also suitable. The results of the tests will be transmitted to the Analytic View.

\subsubsection{Analytic View}

Analytic View is also designed for a future version of this app. It will contain analytics of the data about pilot executed tests and system work. An alert system will be developed for the control room, too. 


\section{IMPLICATIONS FOR PROJECT MANAGEMENT}

SRM concept introduced in this paper together with AR and IoT technologies bring benefits to project management, when compared with the case of not using them:

- Software development can take place even before the development of hardware, which implies a significant reduction in time and resources.

- Reduction of risks in later phases of projects, as many problems can be detected in early phases.

- Presentation of the key features of projects can be provided while the other parts of projects are still under development.

- Examinations can be done repeatedly, and for different functionalities, which enables better inside into initial design and its improvement.

- Development of the most ergonomic design of interactive interface (for example, for training purposes).

- Reduction of costs and increase of efficiency.

\section{CONCLUSION AND FUTURE WORK}

Models are not necessarily simple. In fact, they can be more complex that the real objects or processes they modelled for. For example, a model could involve some experimental parts or some unnecessary parts that would not exist in the final real object or process. Therefore, although the name 'simplified robotic model' could sound as a pleonasm, in fact it is not. In this work the authors found that SRMs can be effectively used to examine or develop a particular feature of a future real object or process. Specifically, for the SRM of centrifuge the authors could successfully design and develop a functional AR/IoT app, as it is described in this paper.

Moreover, working with SRM enables future improvement of the SRM itself and development of advances functionalities of AR/IoT app. The views that are planned for the future version of the app (Pilot/Test View, Analytic View) are going to be thoroughly examined in order to find the most appropriate tests for pilots and analytic insight into the collected data. The authors expect that this approach will bring to effective prediction of pilot behaviour and the centrifuge functionality under high g-forces based on advanced predictive analytics that will be presented in the Analytic View.

The use case presented in this paper showed that AR/IoT app can be successfully designed and developed by using SRM instead of the real object of a concrete project. In addition, the authors concluded that SRM concept with AR/IoT functionalities can be useful for implementation in projects in general, particularly for testing some specific characteristics of projects' subjects.

\section{Acknowledgments}

This paper is a result of project TR-35023 supported by the Ministry of Education and Science of the Republic of Serbia: Development of devices for pilot training and dynamic flight simulation of modern combat aircraft: 3DoF Centrifuge and 4DoF spatial disorientation trainer.

\section{REFERENCES}

Carmigniani, J., Furht, B., Anisetti, M., Ceravolo, P., Damiani, E., \& Ivkovic, M. (2011). Augmented reality technologies, systems and applications. Multimedia Tools and Applications, 51 (1), 341-377, DOI:

https://link.springer.com/article/10.1007 \%2Fs11042-010-0660-6

Cheli, M., Sinapov, J., Danahy, E. E., \& Roger, C. (2018). Towards an Augmented Reality Framework for K-12 Robotics Education, VAM-HRI'2018, March 2018, Chicago, IL USA.

Dodevska, Z., \& Filipović, J. (2018) Application of Augmented Reality in Management and Production in Complex Urban Environment. 1st International Conference on Production in Urban Environments - PRODURB 2018, $17^{\text {th }}$ $19^{\text {th }}$ October 2018, Belgrade, Serbia.

Dodevska, Z., \& Mihić, M. (2018). Augmented Reality and Virtual Reality Technologies in Project Management: What can we Expect? European Project Management Journal, 8 (1), pp. 17-24, DOI: 
https://doi.org/10.18485/epmj.2018.8.1.3, ISSN 2560-4961.

Dodevska, Z., \& Putnik, G. (2018). A Young Researcher's View of Augmented Reality Based on Quantitative Analysis of Articles at Google Scholar in the Last 30 Years. In Proceedings of the $7^{\text {th }}$ International Symposium on Industrial Engineering SIE 2018, Spasojević-Brkić, V., Misita, M., Milanović, D. D., Eds., Industrial Engineering Department, Faculty of Mechanical Engineering, University of Belgrade, Serbia \& Steinbeis Advanced Risk Technologies, Stuttgart, Germany \& Innovation Centre of the Faculty of Mechanical Engineering, University of Belgrade, pp. 259-263, Belgrade, Serbia, $27^{\text {th }}-28^{\text {th }}$ September 2018 , ISBN 978-867083-981-6.

Dodevska, Z. A., Mihić, M. M., \& Manasijević, S. (2018). The Role of Augmented Reality in Defensive Activities, In Proceedings of the 8th International Scientific Conference on Defensive Technologies OTEH 2018, Lisov, M., Ed., Military Technical Institute, pp. 590-593, Belgrade, Serbia, $11^{\text {th }}-12^{\text {th }}$ October 2018, ISBN 9788681123-88-1.

Kvrgic, V. M., Vidakovic, J. Z., Lutovac, M. M., Ferenc, G. Z., \& Cvijanovic, V. B. (2014). A control algorithm for a centrifuge motion simulator. Robotics and Computer-Integrated Manufacturing, 30 (4), pp. 399-412, DOI: https://doi.org/10.1016/j.rcim.2014.01.00 $\underline{2}$
Markov-Vetter, D., Zander, V., Latsch, J., \& Staadt, O. (2015). The Influence of Gravity-adapted Target Resizing on Direct Augmented Reality Pointing under Simulated Hypergravity. In Proceedings of the 10th International Conference on Computer Graphics Theory and Applications (GRAPP-2015), pp. 401411, Berlin, Germany, DOI: https://https:/doi.org/10.5220/000531660 4010411, ISBN: 978-989-758-087-1.

Milgram, P., Takemura, H., Utsumi, A., \& Kishino, F. (1995). Augmented Reality: a class of displays on the reality-virtuality continuum. Proc. SPIE 2351, Telemanipulator and Telepresence Technologies, (21 December 1995); DOI: https://doi.org/10.1117/12.197321

National Instruments (2018). A Practical Guide for Connecting LabVIEW to the Industrial IoT. White Paper, November 2018.

Porter, M. E., \& Heppelmann, J. E. (2014). How Smart, Connected Products Are Transforming Competition. Harvard Business Review, 92 (11), pp. 64-88.

Porter, M. E., \& Heppelmann, J. E. (2017). Why Every Organization Needs an Augmented Reality Strategy. Harvard Business Review, 95 (6), pp. 46-57.

PTC University. Online course: Unpacking the Internet of Things. Heppelmann, J. E. Session: What is the Internet of Things? Why are Businesses Investing in IoT? 